\title{
Knockdown of FOXO6 Inhibits Glycolysis and Reduces Cell Resistance to Paclitaxel in HCC Cells via PI3K/Akt Signaling Pathway
}

This article was published in the following Dove Press journal: OncoTargets and Therapy

\author{
Xixiang Yu' \\ Xixi Gao ${ }^{2}$ \\ Xiaoping $\mathrm{Mao}^{3}$ \\ Zhenjing Shi ${ }^{3}$ \\ Bangxuan Zhu' \\ Linqin $\mathrm{Xie}^{\mathrm{I}}$ \\ Shaodan $\mathrm{Di}^{\mathrm{I}}$ \\ Limin Jin ${ }^{3}$
}

'Wenzhou Medical University, Wenzhou No. 3 Clinical College, Wenzhou, Zhejiang Province, People's Republic of China; ${ }^{2}$ Qingyuan County People's Hospital, Qingyuan, Zhejiang Province, People's Republic of China; ${ }^{3}$ The Third Affiliated Hospital of Wenzhou Medical University, Ruian, Zhejiang Province, People's Republic of China
Correspondence: Limin Jin

The Third Affiliated Hospital of Wenzhou Medical University, No. 108, Wansong

Road, Ruian, Zhejiang Province, People's

Republic of China

Tel +86 I35 $6622 \quad 1074$

Email yinlang25929258258@I63.com
Purpose: Previous studies have reported that FOXO6 is highly expressed in hepatocellular carcinoma (HCC) tissues and is associated with the prognosis of HCC patients. However, little research has been carried out to explore the role of FOXO6 in glycolysis of HCC cells and paclitaxel resistance. Today, along with the increasing incidence and mortality of HCC, chemotherapy resistance of HCC also poses a serious challenge. Therefore, this study was set out to investigate the effect of FOXO6 on glycolysis and cytotoxicity of paclitaxel in HCC cells and its potential mechanism.

Patients and Methods: The levels of FOXO6 mRNA and protein were detected by qRT-PCR and Western blot, respectively. In addition, paclitaxel-resistant cell lines of HCC cells were established, whose activity was assessed by CCK- 8 assay, among which the invasion ability was assessed by Transwell and the apoptosis rate by flow cytometry. What is more, glycolysis levels were evaluated by measuring glucose consumption and lactic acid production, and the protein levels of p-PI3K and p-protein kinase B (Akt) were determined by Western blot.

Results: Compared with normal human hepatocytes, FOXO6 was highly expressed in HCC cells, which was of high real value for HCC. FOXO6 knockdown inhibited the proliferation and invasion and induced apoptosis of HCC cells. In addition, FOXO6 knockdown suppressed glycolysis, reversed resistance to chemotherapy in Hep3B/PTX cells and inactivated $\mathrm{PI} 3 \mathrm{~K}$ and Akt proteins, thus inhibiting the PI3K/Akt signaling pathway. Furthermore, it was found that when activated by $740 \mathrm{Y}-\mathrm{P}, \mathrm{PI} 3 \mathrm{~K} / \mathrm{Akt}$ signaling pathway could resist the effects of FOXO6 knockdown on the cytotoxicity and glycolysis of paclitaxel in HCC cells. Vice versa, inhibition of PI3K/Akt pathway by LY294002 could resist the effect of FOXO6 overexpression on chemotherapy, cytotoxicity and glycolysis of HCC cells.

Conclusion: FOXO6 knockdown can inhibit glycolysis of HCC cells and reduce their resistance to chemotherapy by inhibiting the PI3K/Akt signaling pathway, which may be a new target for the treatment of HCC.

Keywords: FOXO6, PI3K/Akt signaling pathway, hepatocellular carcinoma cells, glycolysis, paclitaxel, drug resistance

\section{Introduction}

As a common malignant tumor, the incidence and mortality of hepatocellular carcinoma (HCC) are on the rise in recent years in parallel with the change of social environment and living habits, posing a serious threat to human life and health. ${ }^{1,2}$ Due to the inconspicuous early symptoms, many HCC patients are only found to be in the advanced stage once diagnosed, depriving them of the opportunity for surgery and treatment. $^{3}$ Currently, hepatic artery perfusion chemotherapy is the mainstream 
treatment method for HCC; however, whose efficacy and prognosis turn out to be poor due to the emergence of resistance of HCC cells. ${ }^{4}$ Therefore, exploring the mechanism of drug resistance of HCC cells is of great clinical significance for the treatment of HCC. Paclitaxel is a pure natural tetracyclic diterpenoid compound extracted from Paclitaxel Short Leaf. As a first-line chemotherapeutic agent for a variety of tumors, it can inhibit the depolymerization of tubulin to block the mitosis of tumor cells and ultimately induce apoptosis of tumor cells and eventually induce the apoptosis of tumor cells. ${ }^{5,6}$ Whereas, paclitaxel displayed no obvious effect on HCC, and its drug resistance mechanism also has not been investigated.

Transcription factor Forkhead box (FOXO), which includes FOXO1, FOXO3, FOXO4, and FOXO6 genes, exerts a marked effect on cell metabolism, death, and oxidative stress. ${ }^{7}$ Previous studies ${ }^{8}$ exhibited that FOXOs could affect insulin resistance by inhibiting the PIK3/AKt signaling pathway. Others ${ }^{9}$ revealed the high expression of FOXO1 in Paclitaxel-resistant ovarian cancer cells, suggesting a close link between FOXOs and chemotherapy resistance. FOXO6, as a member of the FOXO family, is expressed in mammals and is considered to play a vital part in glucose metabolism and energy balance of tumor cells like FOXO1. ${ }^{10}$ While its difference from FOXO1 lies in that, FOXO6 was previously thought to be the least distinctive member of FOXOs, mainly acting significantly on liver, skeletal muscle and brain. ${ }^{11}$ In addition, it was reported that FOXO6 was highly expressed in HCC and associated with poor prognosis of HCC patients. ${ }^{12}$ However, no research has been conducted on the effect and underlying mechanism of FOXO6 in HCC cells. Integrated with the above studies, we reasonably speculated that FOXO6 may be related to paclitaxel resistance and glycolysis in HCC cells.

Therefore, the biological role and mechanism of FOXO6 in paclitaxel resistance of HCC cells were investigated in the present study, aiming to provide new molecular directions and preventing resistance to chemotherapy for the treatment of HCC.

\section{Materials and Methods}

\section{Clinical Specimens}

Seventy-one patients who underwent hepatectomy in the Third Affiliated Hospital of Wenzhou Medical University hospital from February 2016 to February 2018 were collected, with an average age of $(61.33 \pm 4.26)$ years. Paired HCC tissues and adjacent tissues normal tissue were obtained intraoperatively from all the study subjects and stored in liquid nitrogen tanks after the consent of the patients. The baseline data are shown in Table 1. Inclusion criteria: Patients pathologically diagnosed with HCC for the first time were included in this study. In contrast, the exclusion criteria were as follows: Patients who received chemoradiotherapy, combined with other malignant tumors, severe renal dysfunction, or severe infectious disease, and those who refused to provide experimental specimens were excluded. All patients and their families agreed to participate in the experiment and signed the informed consent. All procedures performed in studies involving human participants were in accordance with the ethical standards of the Third Affiliated Hospital of Wenzhou Medical University ethics committee and with the 1964 Helsinki declaration and its later amendments or comparable ethical standards.

\section{Institutional Review Board Statement}

This study was reviewed and approved by the Third Affiliated Hospital of Wenzhou Medical University Ethics Committee.

\section{Materials and Reagents}

Human HCC cell lines HepG2, Huh-7, Hep3B, BEL-7402 and human normal hepatocyte line HL-7702 were purchased

Table I General Information

\begin{tabular}{|l|l|}
\hline Data & HCC Patients (n=7 I) \\
\hline Gender & \\
Male & $48(67.61)$ \\
Female & $23(32.39)$ \\
Age (years) & $61.33 \pm 4.26$ \\
BMI (Kg/m $\left.{ }^{2}\right)$ & $22.75 \pm 1.53$ \\
\hline Pathological type & \\
Hepatocellular carcinoma & $31(43.66)$ \\
Cholangiocarcinoma & $24(33.80)$ \\
Mixed cancer & $16(22.54)$ \\
\hline Pathological staging & \\
I & $22(30.99)$ \\
II & $29(40.85)$ \\
III & $20(28.17)$ \\
\hline Differentiation degree & \\
High & $21(29.58)$ \\
Moderate & $22(30.99)$ \\
Low & $28(39.44)$ \\
\hline Metastasis & $32(45.07)$ \\
With & $39(54.93)$ \\
Without & \\
\hline
\end{tabular}


from Beinachuanglian Biotechnology Research Institute, China, subordinate agent of ATCC, with the number of 338070, 337690, 337952, 338237, 351907, respectively. Paclitaxel P132500 was acquired from TRC Canada, qRTPCR and reverse transcription kit were from TransGen Biotech, Beijing, China, with the corresponding number of AQ201-01, AQ202-01. Double luciferase reporter gene detection kit was obtained from Beijing Biolab Technology Co., Ltd., KFS303-TFX, and CCK-8 kit from Wuhan MSK Biotechnology Co., Ltd. Transwell Kit, with the number of FK-lk019, was acquired from Shanghai Fanke Biotechnology Co., Ltd., and both PBS, bovine fetal serum (FBS) from Gibco Co., USA. Trizol reagent was obtained from Thermo Fisher Scientific (China) Co., Ltd., with the number of 15596018, while RIPA, BCA protein reagent kit were purchased from Thermo Scientific Co., USA. Annexin V-FITC/PI cell apoptosis reagent kit (Thermo Fisher Scientific (China) Co., Ltd., V13241), FOXO6, p-Akt, p-PI3K, Caspase-3, Bax, Bcl-2, Glut1, HK 2 and LDH-A and $\beta$-Actin antibodies (Cell Signaling Technology Company) were applied in this study. Besides, goat antirabbit IgG antibody (Beijing Sino Biological, SSA004200), and ECL developer (Thermo Company) were employed. Still, PCR (ABI Company, USA, 7500), glucose determination kit and lactic acid determination test SigmaAldrich (GAHK 20-1KT, MAK064-1KT), 740Y-P (Selleck Chemicals, S7865), LY294002 (Selleck Chemicals, S1105) were adopted in this study. All the primers were designed and synthesized by Sangon Biotech (Shanghai) Co., Ltd.

\section{Cell Culture and Transfection}

HCC cell linesHepG2, Huh-7, Hep3B, BEL-7402human normal hepatocyte line HL-7702 were cultured in Dulbecco's Modified Eagle's Medium (DMEM) containing $10 \% \mathrm{PBS}, 2 \mathrm{~mm}$ penicillin and streptomycin at $37^{\circ} \mathrm{C}$ with $5 \% \mathrm{CO}_{2}$. When the cell adherent growth and fusion reached $85 \%, 25 \%$ trypsin was added for digestion. After that, each cell line was placed in the medium for further culture, passage and finally for FOXO6 detection. Next, HepG2 and Hep3B cells were selected for transfection and then targeted inhibition of FOXO6 RNA (si-FOXO6), negative control RNA (Si-NC), targeted overexpression of FOXO6 RNA (sh-FOXO6), negative control RNA (Sh-NC) were established. Lipofectamine ${ }^{\mathrm{TM}} 2000$ kit was employed for cell transfection, and the procedures were carried out in strict accordance with the kit instructions.

\section{Culture of Resistant Cell Lines}

Hep3B cells at logarithmic phase were cultured in DMEM medium containing $10 \% \mathrm{PBS}, 2 \mathrm{~mm}$ penicillin and streptomycin, and $0.05 \mu \mathrm{mol} / \mathrm{L}$ paclitaxel was added in the medium for $24 \mathrm{hrs}$ at $37^{\circ} \mathrm{C}$ and $5 \% \mathrm{CO}_{2}$ before a triple rinse with PBS. Then, fresh culture medium was added to continue the culture until the cells resumed logarithmic growth. After that, another $0.05 \mu \mathrm{mol} / \mathrm{L}$ paclitaxel was added for screening, followed by repeated screening with paclitaxel at the concentrations of $0.15 \mu \mathrm{mol} / \mathrm{L}, 0.155$ $\mu \mathrm{mol} / \mathrm{L}$, and $0.25 \mu \mathrm{mol} / \mathrm{L}$ for 2 times. Upon the completion of the final screening, Hep3B/PTX cells were cultured in a culture medium containing $0.01 \mu \mathrm{mol} / \mathrm{L}$ paclitaxel to maintain drug resistance and FOXO6 intervention.

\section{Real-Time Quantitative PCR}

Firstly, the total RNA in tissues and cells was extracted with Trizol reagent. Then, $5 \mu \mathrm{g}$ of the total RNA was taken for cDNA reverse transcription according to the kit instructions, followed by the amplification of the synthesized cDNA $(1 \mu \mathrm{g})$. The amplification system: Upstream and downstream primers: $0.4 \mu \mathrm{L}$, respectively, $2 \times$ TransTaq $^{\circledR}$ Tip Green qPCR SuperMix: $10 \mu \mathrm{L}$, Passive Reference Dye (50X): $0.4 \mu \mathrm{L}$, and Nuclease-free Water was added to complete to $20 \mu \mathrm{L}$. With $\beta$ Actin as the internal reference for FOXO6, $2^{-\triangle \Delta \text { ct }}$ was employed to analyze the data. Primer sequence: FOXO6 upstream primer: 5'-GGCCGCGCTCGTGTACC-3', downstream primer: 5'-TACACGAGCGCG GCCG-3'; $\beta$-Actin upstream primer: 5'-CTACGTCGCCCTGGACTTCGAGC3', downstream primer: 5'-GATG GAGC CGCC GATC CACA CGG-3'.

\section{Western Blot Detection}

RIPA LYSIS was used to lyse the cells and extract the total protein, and then the protein concentration was detected by BCA assay, whose procedures were as follows: The protein concentration was adjusted to $4 \mathrm{ug} / \mu \mathrm{L}$, electrophoretically separated by $12 \%$ SDS-PAGE before transferring to PVDF membrane, and then sealed by $5 \%$ skim milk powder for 2hrs. Next, FOXO6 (1:500), p-Akt (1:500), p-PI3K (1:500), Caspase-3 (1:500), Bax (1:500), Bcl-2 (1:500), Glut1 (1:500), HK2 (1:500), LDH-A (1:500) and $\beta$-Actin primary antibody $(1: 1000)$ were added and sealed overnight at $4^{\circ} \mathrm{C}$. After that, the membrane was washed to remove the primary antibody, followed by the adding of horseradish peroxidase-labeled goat anti-rabbit secondary antibody $(1: 1000)$, then incubated at $37^{\circ} \mathrm{C}$ for $1 \mathrm{~h}$, and rinsed with 
PBS for 3 times, 5 min each. And then developed in a dark room, dried the extra liquid on the membrane with filter paper, and developed with ECL luminescence. Finally, the protein bands were scanned and analyzed in Quantity One software for gray value, where the relative protein expression=gray value of the target protein bands/gray value of the $\beta$-Actin protein bands.

\section{Cell Proliferation Experiment}

CCK-8 kit was adopted to evaluate cell proliferation. Cells were collected $48 \mathrm{hrs}$ after transfection and diluted to $3 \times 10^{4}$ cells $/ \mathrm{mL}$. Then, they were inoculated in 96-well plates, $100 \mu \mathrm{L}$ cells per well, and cultured at $37^{\circ} \mathrm{C}$ with $5 \% \mathrm{CO}_{2}$. At $0 \mathrm{~h}, 24 \mathrm{~h}, 48 \mathrm{~h}$ and $72 \mathrm{~h}$ after adherent growth, CCK 8 solution of 10 mics was added to each well, and the culture was continued for $2 \mathrm{~h}$ in an incubator with $5 \% \mathrm{CO}_{2}$ at $37^{\circ} \mathrm{C}$ after addition of a reagent. Then, the OD value was measured at $450 \mathrm{~nm}$ with a microplate reader to detect cell proliferation and plot growth curve. The experiment was repeated three times.

\section{Evaluation of Paclitaxel IC50 in HCC Cells}

Cells were inoculated in 96-well plates at the density of $1 \times 10^{5}$ per well, and $0.2 \mathrm{~mL}$ paclitaxel with concentrations of $0.1 \mu \mathrm{mol} / \mathrm{L}, 0.5 \mu \mathrm{mol} / \mathrm{L}, 1.0 \mu \mathrm{mol} / \mathrm{L}, 1.5 \mu \mathrm{mol} / \mathrm{L}, 2 \mu \mathrm{mol} / \mathrm{L}$ and $2.5 \mu \mathrm{mol} / \mathrm{L}$ were added, respectively, before inoculation for $48 \mathrm{~h}$. Then, the culture medium was renewed, followed by the adding of CCK- 8 solution to each well and then cultured in an incubator for $2 \mathrm{~h}$. The experiment was repeated three times. And then the absorbance of each hole was measured using SpectraMax M5 spectrometer at a wavelength of $450 \mathrm{~nm}$. Finally, the IC50 of paclitaxel on Hep3B/PTX cells was calculated according to the cell survival rate (GraphPad prism).

\section{Cell Apoptosis Experiment}

The transfected cells were digested with $0.25 \%$ trypsin and rinsed with PBS twice and then $100 \mu \mathrm{L}$ binding buffer was added to configure into $1 * 10^{6} / \mathrm{mL}$ suspension. After that, Annexin V-FITC and PI were added successively, incubated in the dark at room temperature for 5 mins, and detected by the FACSVerse flow cytometry system. The experiment was repeated three times to get the average value.

\section{Cell Invasion Experiment}

Cell invasion was assessed by Transwell. Firstly, $200 \mu \mathrm{L}$ DMEM culture solution containing $1 \times 10^{5}$ cells was added to the upper chamber, and $500 \mathrm{~mL}$ DMEM containing 20\% FBS was added to the lower chamber and cultured at $37^{\circ} \mathrm{C}$ for $48 \mathrm{~h}$. The stroma and cells failed to cross the membrane surface were wiped and rinsed with PBS for 3 times, followed by a 10-min' fixing with paraformaldehyde, and then washed with doublesteamed water for 3 times. After drying, they were stained with $0.1 \%$ Crystal Violet Stain Solution for $10 \mathrm{~min}$. Finally, the cell invasion was observed using a microscope.

\section{Glucose Consumption and Lactic Acid Content}

The collected cells were inoculated in 6-well plates at the density of $3 \times 10^{5}$ per well, cultured at $37^{\circ} \mathrm{C}$ and $5 \% \mathrm{CO}_{2}$ for $48 \mathrm{~h}$. Then, the culture medium was collected for the determination of glucose consumption and lactic acid production strictly followed the corresponding kit instructions.

\section{Statistical Analysis}

The collected data were statistically analyzed using SPSS20.0 software and plotted by GraphPad 7 in the current study. An independent sample $t$-test was adopted for intra-group comparison and One-way ANOVA was employed for multi-group comparisons. LSD- $t$ test was applied for posthoc pairwise comparison, MANOVA of repeated measures was used for multiple time points, and Bonferroni for post-test verification. A statistically significant difference was assumed at $\mathrm{P}<0.05$.

\section{Results}

\section{Expression and Diagnostic Value of FOXO6 in HCC}

RT-PCR and Western Blot assays showed that the mRNA and protein levels of FOXO6 in HCC tissues were significantly up-regulated. The ROC curve analysis demonstrated that both the mRNA and protein of FOXO6 were more than 0.9 in the diagnosis of HCC, which indicated high diagnostic value (Figure 1).

\section{Effects of FOXO6 on Proliferation, Invasion and Apoptosis of HCC Cells}

The expression of FOXO6 in HCC cell lines HepG2, Huh7, Hep3B and BEL-7402 was significantly higher than that in human normal hepatocyte line HL-7702 $(\mathrm{P}<0.05)$. The FOXO6 expression in the HepG2 and Hep3B transfected with Si-FOXO6 cells was significantly down-regulated compared with those transfected with Si-NC cells, and was significantly up-regulated in the Sh-FOXO6 transfected cells than those of the Sh-NC group $(\mathrm{P}<0.05)$. No 

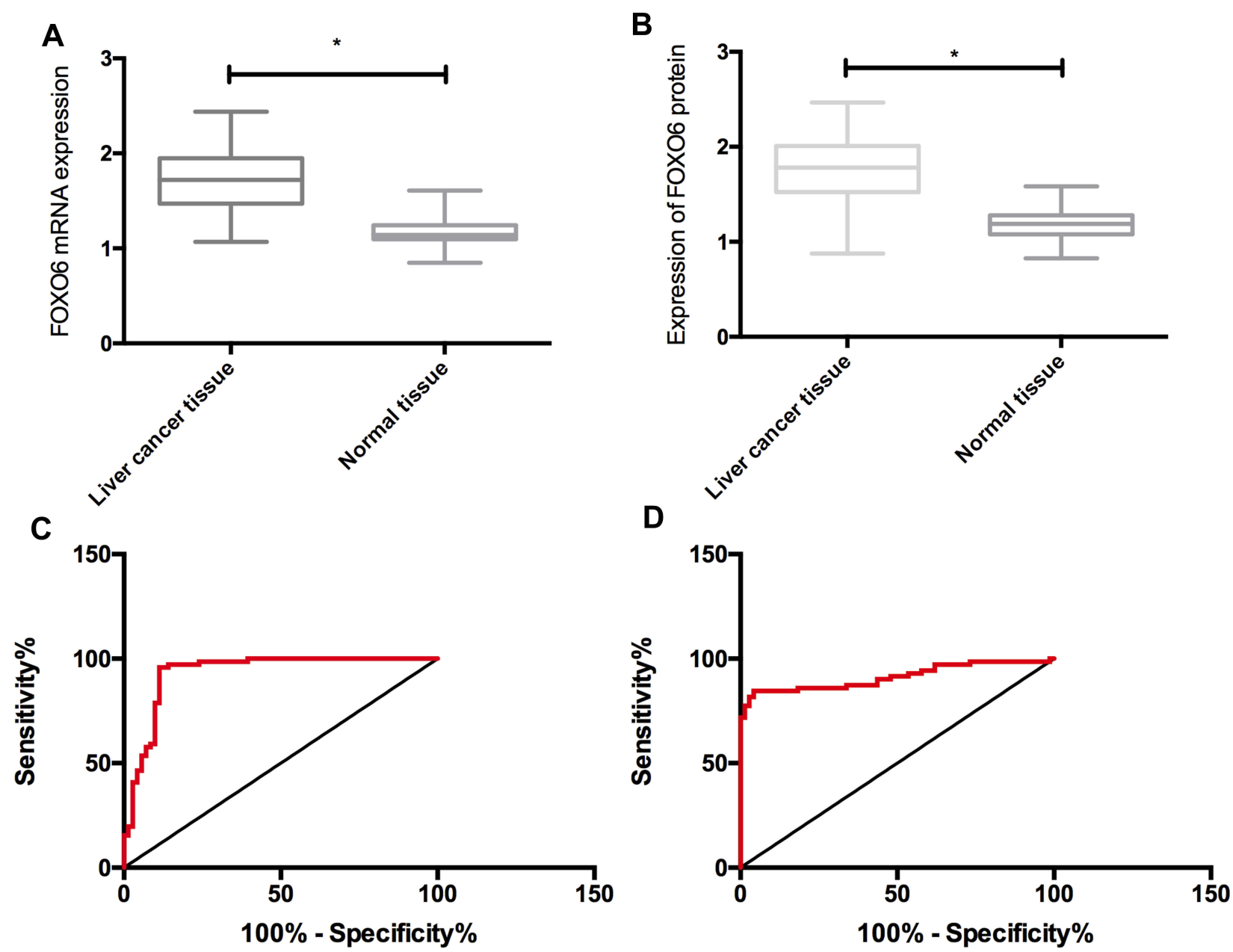

Figure I Expression and clinical significance of FOXO6 in HCC. (A) Expression of FOXO6 mRNA in HCC. (B) Expression of FOXO6 protein in HCC. (C) Diagnostic value of FOXO6 mRNA in HCC. (D) Diagnostic value of FOXO6 protein in HCC. *Indicates $\mathrm{P}<0.05$.

significant difference was observed in FOXO6 expression between the Si-NC and Sh-NC groups $(\mathrm{P}>0.05)$. As to the biological functions of cells in the two groups, the cells transfected with Si-FOXO6 presented markedly decreased proliferation and invasion ability, significantly increased apoptosis rate, notably decreased expression of Bcl-2 and remarkably elevated Caspase-3 and Bax protein expressions than the Si-NC group $(\mathrm{P}<0.05)$. While compared with the Sh-NC group, the proliferation and invasion ability of the Si-FOXO6 transfected cells increased significantly, the apoptosis rate reduced notably $(\mathrm{P}<0.05)$, the Bcl-2 expression elevated markedly, and Caspase- 3 and Bax protein expressions decreased remarkably $(\mathrm{P}<0.05)$ (Figure 2).

\section{Effects of FOXO6 on Paclitaxel Toxicity}

Paclitaxel significantly inhibited the proliferation of HepG2 and Hep3B cells $(\mathrm{P}<0.05)$ in a dose-dependent manner. The IC50 of Hep3B/PTX cells to paclitaxel was significantly higher than that of HepG2 and Hep3B cells $(\mathrm{P}<0.05)$. In addition, down-regulated FOXO6 expression could significantly increase the sensitivity of HepG2 and Hep3B cells, reverse the resistance of Hep3B/PTX cells, and reduce the IC50 of cells to paclitaxel $(\mathrm{P}<0.05)$. Vice versa, up-regulation of FOXO6 could reduce the sensitivity of HepG2 and Hep3B cells, enhance the resistance of Hep3B/PTX cells, and up-regulate the IC50 of cells to paclitaxel $(\mathrm{P}<0.05)$ (Figure 3$)$.

\section{Effects of FOXO6 on Glycolysis and PI3K/} Akt Signaling in HCC Cells

The HepG2 and Hep3B cells transfected with SiFOXO6 displayed significantly inhibited glycolysis level $(\mathrm{P}<0.05)$, markedly down-regulated expressions of Glut1, HK2 and LDH-A protein $(\mathrm{P}<0.05)$, and notably decreased expressions of $\mathrm{p}-\mathrm{Akt}$ and $\mathrm{p}-\mathrm{PI} 3 \mathrm{~K}(\mathrm{P}<0.05)$ 

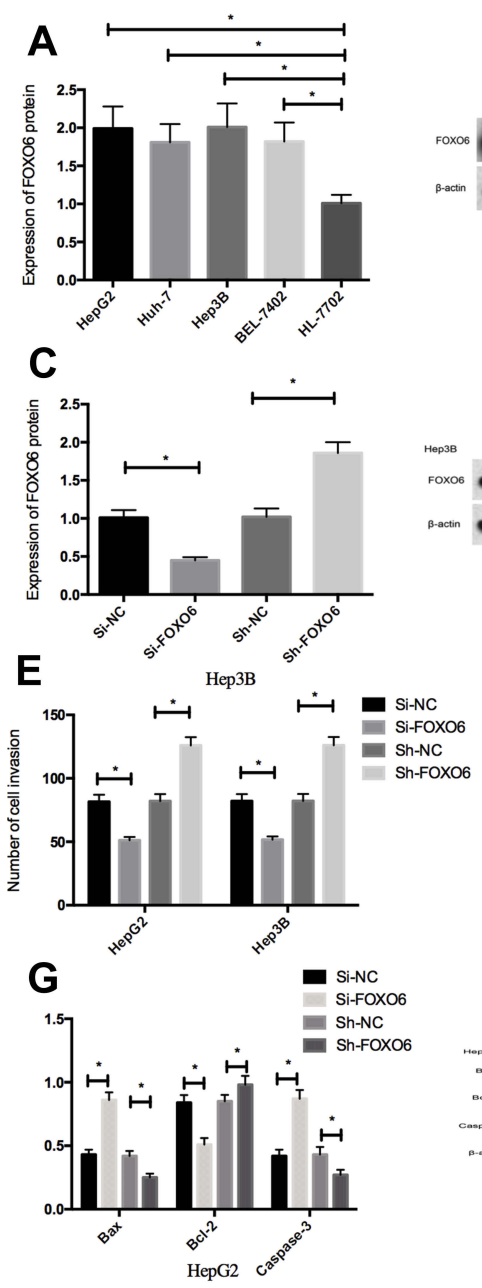

B
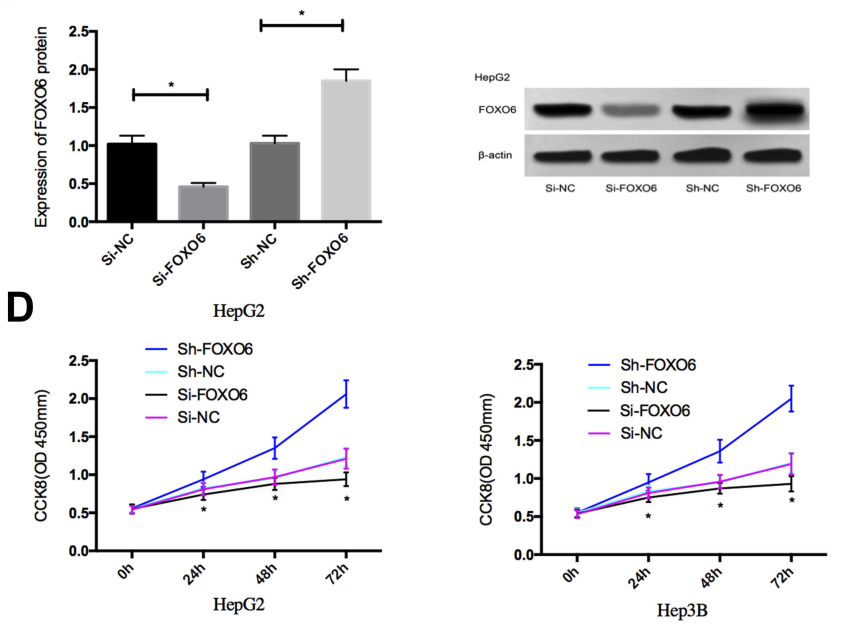

$\mathbf{F}$

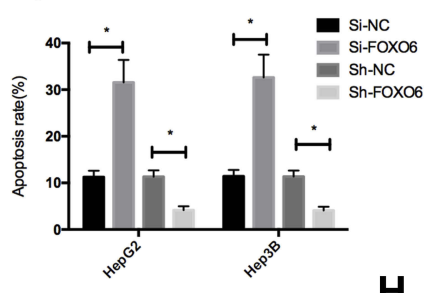

H

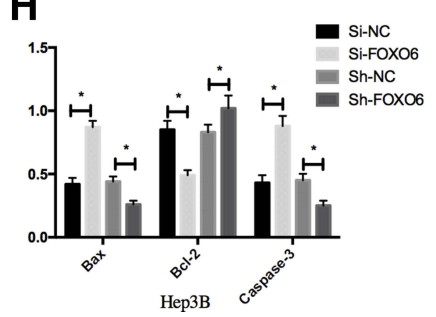

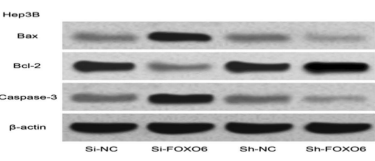

Figure 2 Effects of FOXO6 on proliferation, invasion and apoptosis of HCC cells. (A) FOXO6 expression in HCC cells. (B) FOXO6 expression in transfected HepG2 cells. (C) FOXO6 expression in transfected Hep3B cells. (D) Proliferation ability of transfected HepG2 and Hep3B cells. (E) Invasion ability of transfected HepG2 and Hep3B cells. (F) Apoptosis rates of transfected HepG2 and Hep3B cells. (G) Expression of apoptosis-related proteins in transfected HepG2 cells. (H) Expression of apoptosis-related proteins in transfected Hep3B cells. *Indicates $\mathrm{P}<0.05$.

than cells in the Si-NC group. While compared with the Sh-NC group, the glycolysis of HepG2 and Hep3B cells transfected with Sh-FOXO6 elevated significantly $(\mathrm{P}<0.05)$, the expressions of Glut1, HK2 and LDH-A protein up-regulated markedly $(\mathrm{P}<0.05)$, and meanwhile, the expressions of p-Akt and p-PI3K increased remarkably $(\mathrm{P}<0.05)$ (Figure 4).

\section{Effects of Activating PI3K/Akt Signaling Pathway on HCC Cells After FOXO6 Knockdown}

After 48h treatment of untransfected HepG2 and Hep3B cells with $10 \mu \mathrm{L} 740 \mathrm{Y}-\mathrm{P}$ (an activator of PI3K/Akt pathway), the expression of p-Akt and p-PI3K in HepG2 and Hep3B cells treated with 740Y-P increased significantly than those without $(\mathrm{P}<0.05)$, suggesting that $740 \mathrm{Y}-\mathrm{P}$ could activate the PI3K/Akt signaling pathway. The cells transfected with Si-NC and Si-FOXO6 were then exposed to $5 \mu \mathrm{L} 740 \mathrm{Y}-\mathrm{P}$ for $48 \mathrm{~h}$, and then to paclitaxel at a concentration of $1.5 \mu \mathrm{mol} / \mathrm{L}$ for $48 \mathrm{hrs}$. The results showed that Si-FOXO6 could further enhance the inhibition of paclitaxel on the proliferation, invasion and glycolysis of HepG2 and Hep3B cells, and reverse the apoptosis of HepG2 and Hep3B cells induced by paclitaxel, but this promotion effect was inhibited after adding 740Y-P. What is more, it was observed that Si-FOXO6 could further stimulate paclitaxel to increase the expression of Caspase- 3 and Bax, and decrease the expression of Bcl-2, Glut1, HK 2 and LDH-A in Hep3B cells, which was consistent with the phenotype. However, after adding 740Y-P, the promotion effect was inhibited (Figure 5). 


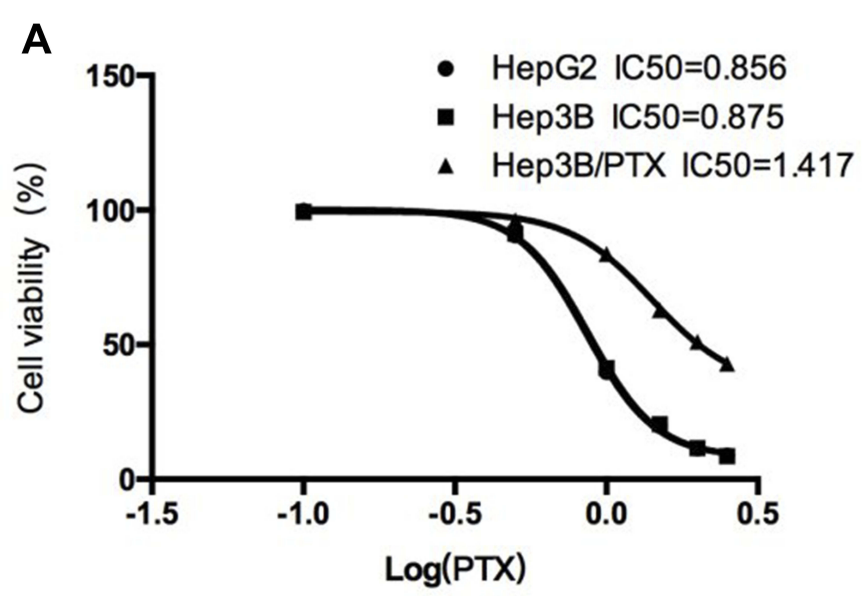

C

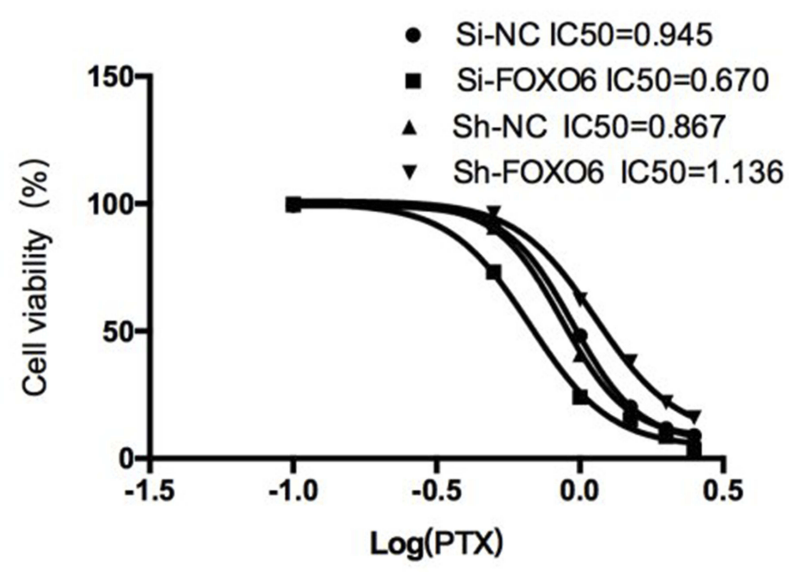

Hер3B

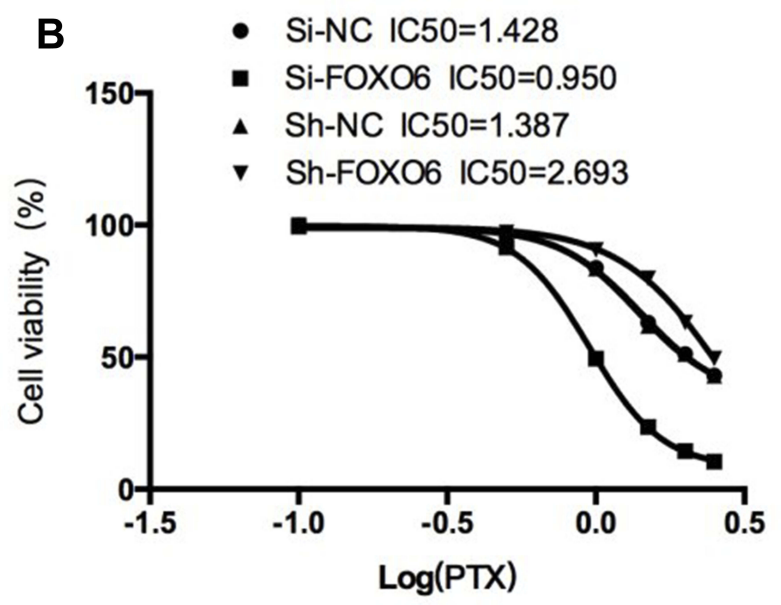

Hep3B/PTX

D

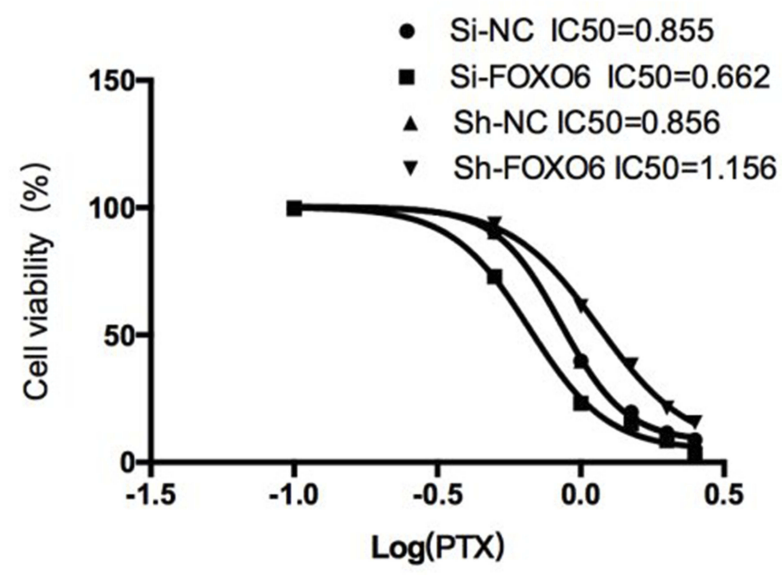

HepG2

Figure 3 Effects of FOXO6 on paclitaxel toxicity. (A) IC50 of paclitaxel on HepG2, Hep3B and Hep3B/PTX cells. (B) IC50 of paclitaxel on HepG2 cells after FOXO6 regulation. (C) IC50 of paclitaxel on Hep3B cells after FOXO6 regulation. (D) IC50 of paclitaxel on Hep3B/PTX cells after FOXO6 regulation.

\section{Inhibition of PI3K/Akt Signaling Pathway on HCC Cells After Overexpression of FOXO6}

After $48 \mathrm{~h}$ treatment of HepG2 and Hep3B cells with $5 \mu \mathrm{L}$ LY294002 (an inhibitor of PI3K/Akt pathway), the expressions of p-Akt and p-PI3K in HepG2 and Hep3B cells were found significantly reduced compared with those without LY294002 treatment $(\mathrm{P}<0.05)$. Then, the cells transfected with Sh-FOXO6 were exposed to $5 \mu \mathrm{L}$ LY294002 for $48 \mathrm{~h}$ and then to a concentration of 0.5 $\mu \mathrm{mol} / \mathrm{L}$. The results revealed that Sh-FOXO6 could restore the inhibition of paclitaxel on the proliferation, invasion and glycolysis of HepG2 and Hep3B cells, and inhibited their apoptosis induced by paclitaxel, but the inhibition effect was reversed after addition LY294002. It was also observed that Sh-FOXO6 could inhibit the expression of Caspase-3 and Bax proteins in HepG2 and Hep3B cells induced by paclitaxel, while the expression of $\mathrm{Bcl}-2$, Glut1, HK 2 and LDH-A protein decreased, which was consistent with the phenotype (Figure 6).

\section{Discussion}

Recent years have witnessed the increase in the incidence of HCC with the change of social environment. ${ }^{13}$ Currently, chemotherapy resistance remained the main factor leading to poor therapeutic efficacy and poor prognosis in HCC patients, so it is of great clinical significance to explore the drug resistance mechanism of HCC cells. ${ }^{14}$ In our study, FOXO6 was first demonstrated to be associated with glycolysis and paclitaxel resistance in $\mathrm{HCC}$ 


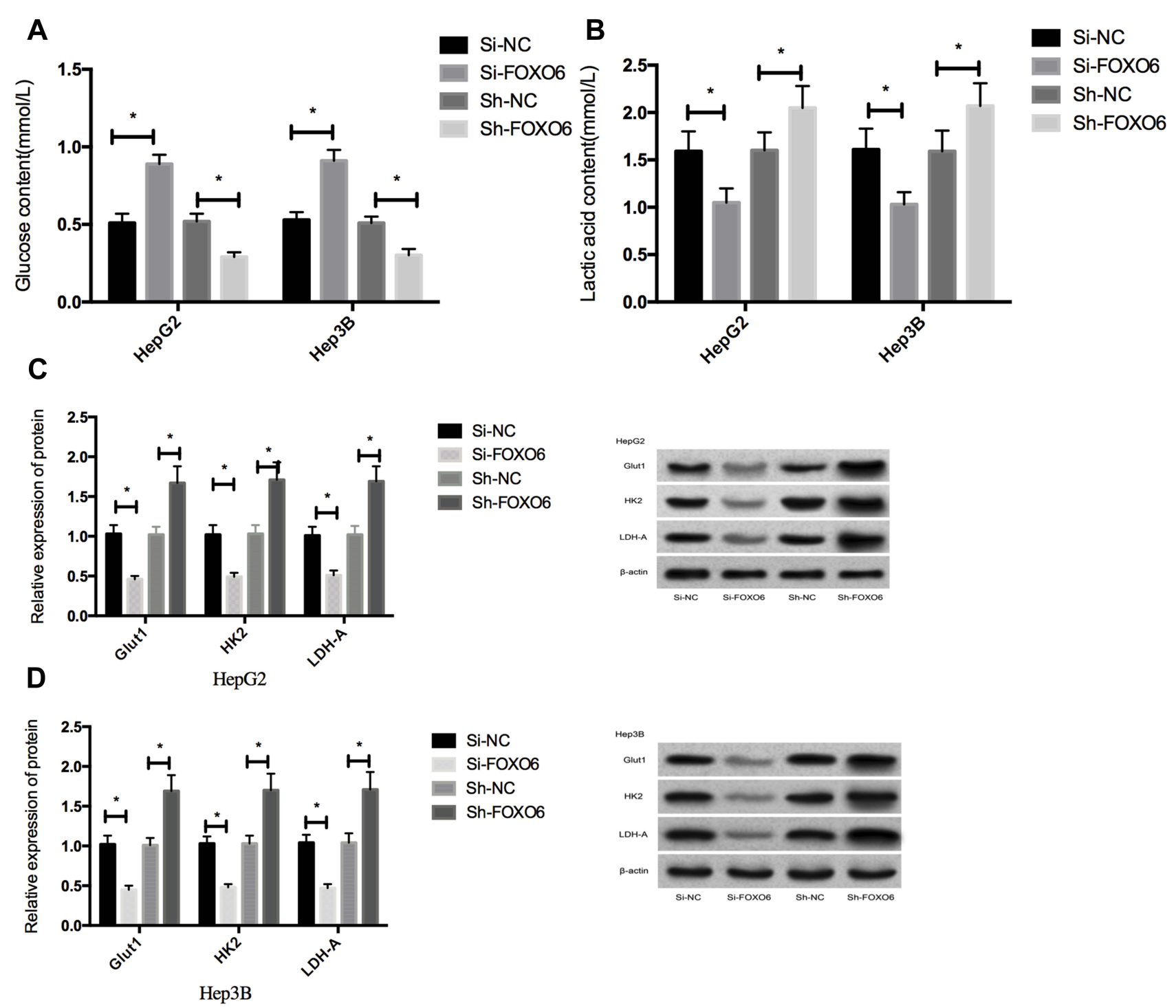

E
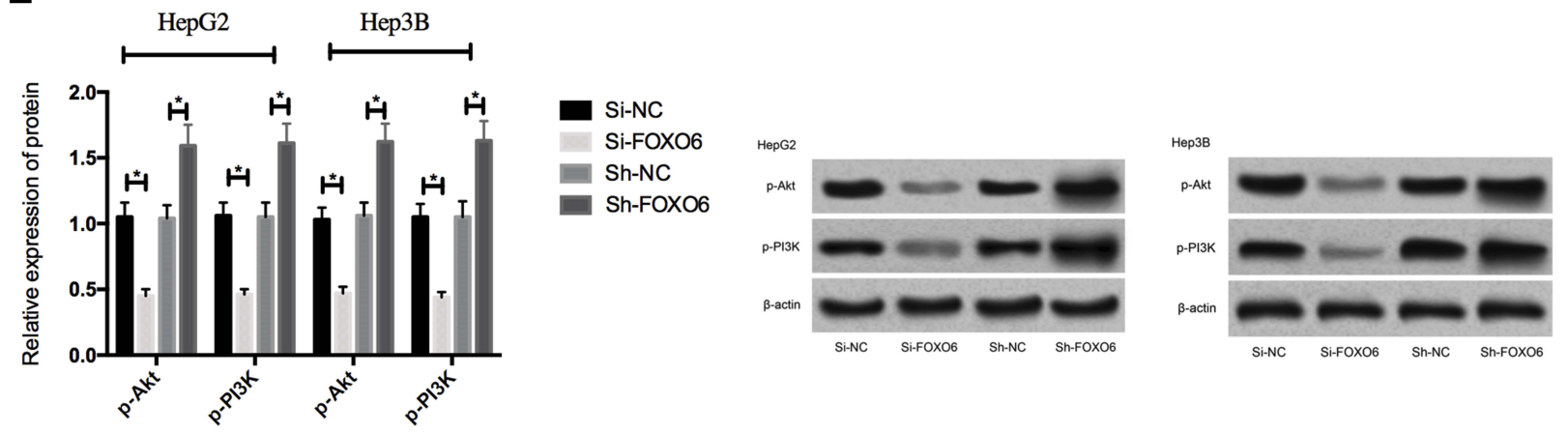

Figure 4 Effects of FOXO6 on glycolysis and PI3K/Akt signaling pathway in HCC cells. (A) Glucose content in the culture medium after transfection. (B) Lactic acid content in the culture medium after transfection. (C) Expression of glycolysis-related proteins in HepG2 cells. (D) Expression of glycolysis-related proteins in Hep3B cells. (E) Expressions of p-Akt and p-PI3K in HCC cells after transfection. *Indicates $\mathrm{P}<0.05$.

cells, whose mechanism may be achieved by affecting the PI3K/Akt signaling pathway.

As a gene with specific functions, the expression of FOXO varies in different parts of tissues. For example,
FOXO1 mostly expressed itself in liver tissues and adipose tissues, while FOXO4 mainly manifested in skeletal muscles and FOXO6 in liver tissues. ${ }^{15,16}$ The current study found that the expression of FOXO6 in HCC tissues was 


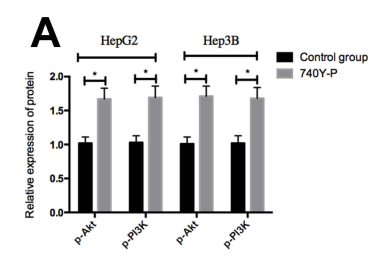

D
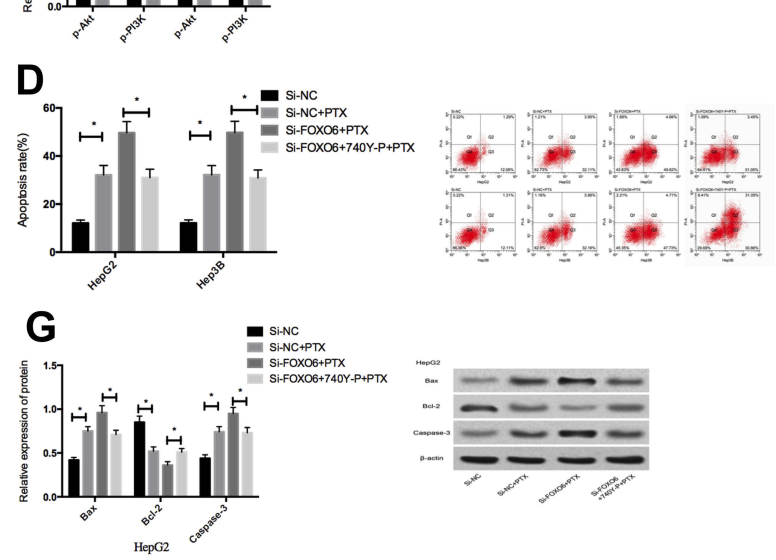

I
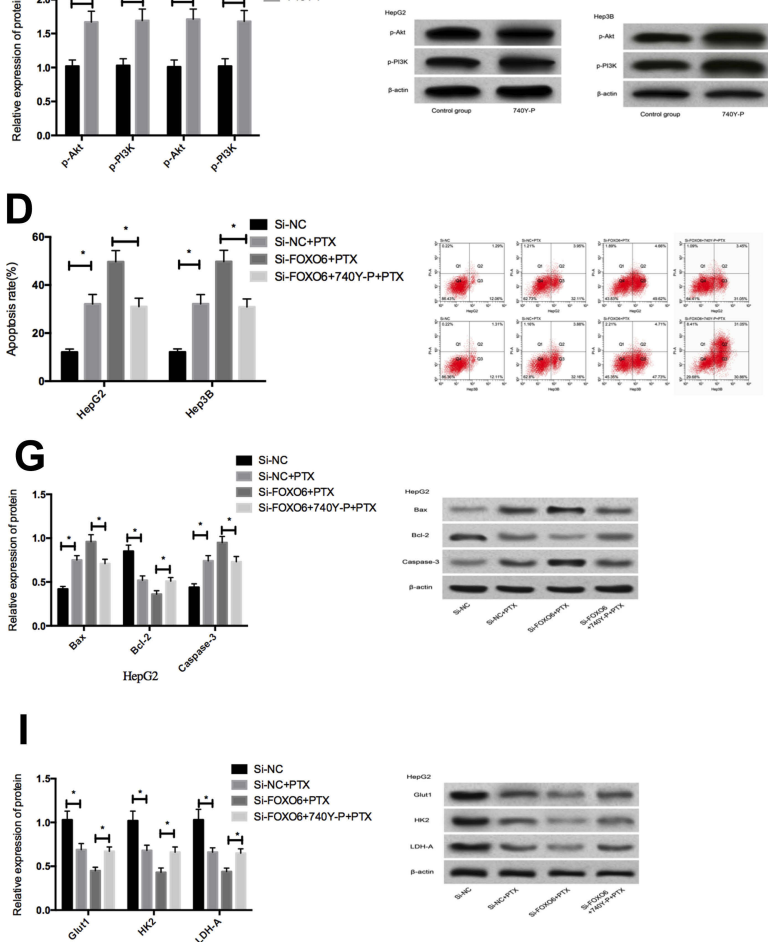

E
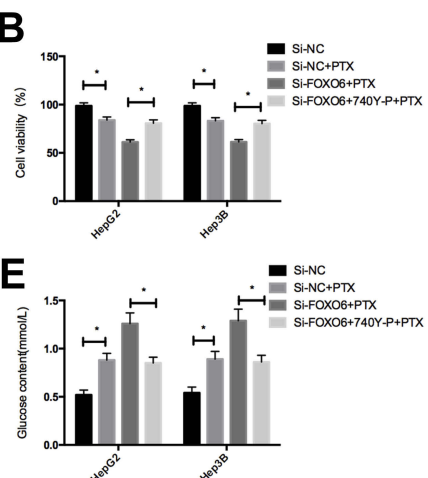

H

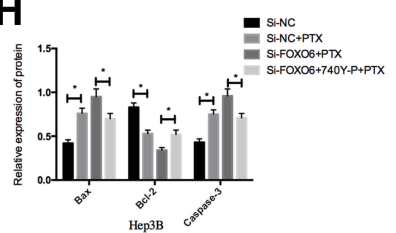

$\mathbf{J}$

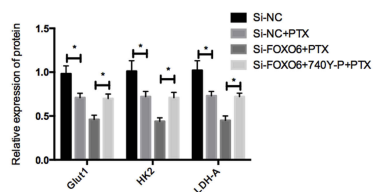

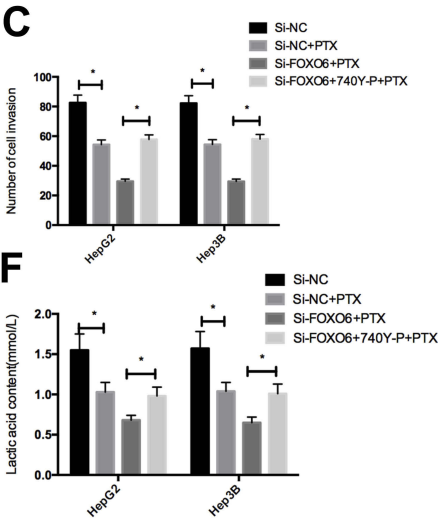
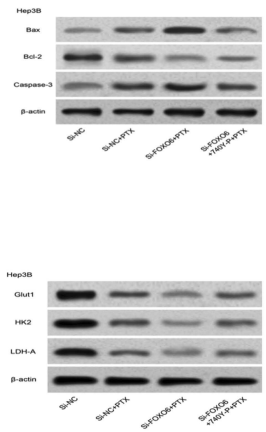

Figure 5 Effects of activating PI3K/Akt signaling pathway on HCC cells after FOXO6 knockdown. (A) Expressions of p-Akt and p-PI3K proteins in HCC cells after activation of the PI3K/Akt signaling pathway. (B) Comparison of cell viability. (C) Comparison of cell invasiveness. (D) Comparison of apoptosis rates. (E) Comparison of glucose contents in the culture medium. (F) Comparison of lactic acid content in the culture medium. (G) Expression of apoptosis-related proteins in HepG2 cells. (H) Expression of apoptosis-related proteins in Hep3B cells. (I) Expression of glycolysis-related proteins in HepG2 cells. (J) Expression of glycolysis-related proteins in Hep3B cells. *Indicates $\mathrm{P}<0.05$.

significantly higher than that in adjacent tissues and normal hepatocytes, which was consistent with previous studies. Further interfering with the expression of FOXO6 in HCC cells revealed that downregulation of FOXO6 expression could significantly inhibit the proliferation and invasion and promote the apoptosis of HCC cells, while the opposite effects were achieved by upregulating FOXO6 expression, suggesting that FOXO6 played an oncogenic role in HCC cells. Studies in the past indicated that FOXO6 acted as a pro-cancer factor in other tumors. For example, studies ${ }^{17}$ demonstrated that inhibition of FOXO6 expression in breast cancer could significantly block the growth cycle of breast cancer cells. Then, in order to investigate the effect of FOXO6 on paclitaxel resistance in HCC cells, Hep3B cell lines, which were more sensitive to paclitaxel, ${ }^{18}$ were selected to establish paclitaxel-resistant cell lines in this study. Firstly, the IC50 of paclitaxel to HepG2, Hep3B and Hep3B/PTX cells was compared, and the results showed that the IC50 of Hep3B/PTX cells was significantly higher than that of HepG2 and Hep3B cells, indicating that Hep3B/PTX had obvious resistance to paclitaxel. And then the IC50 of
HepG2, Hep3B and Hep3B/PTX cells treated with FOXO6 was compared. The results revealed that downregulated FOXO6 expression could significantly increase the sensitivity of HepG2 and Hep3B cells, reverse the resistance of Hep3B/PTX cells, and reduce the IC50 of cells to paclitaxel, while up-regulated FOXO6 had the opposite effect. This is the first time that we have demonstrated that downregulation of FOXO6 expression in HCC cells can reverse the resistance of HCC cells to paclitaxel. It was well established that ${ }^{19}$ the FOXO family regulated the several transcription targets that may affect tumor cells, response to drugs and sensitivity to drugs. Reports over the past focused more about FOXO1, FOXO3 and FOXO4, and it was believed that the fusion DNA generated between the transcription domain of FOXO and the DNA binding domain of other spouses can affect the development of tumors by fusing proteins, and the abnormal proteins after fusion can affect the transcriptional efficiency, which will lead to cell response to drugs. ${ }^{20,21}$ However, the mechanism of FOXO6 in tumor response to chemotherapeutic drugs remained a subject of investigation. 


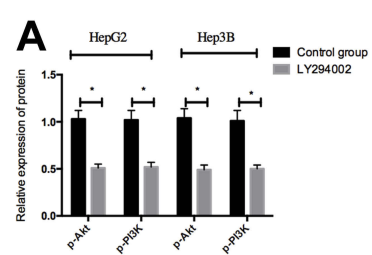

D

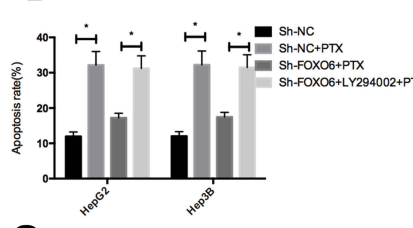

G

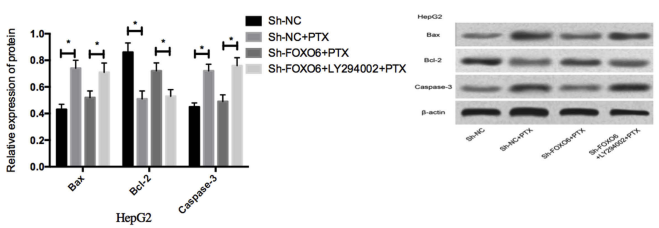

I

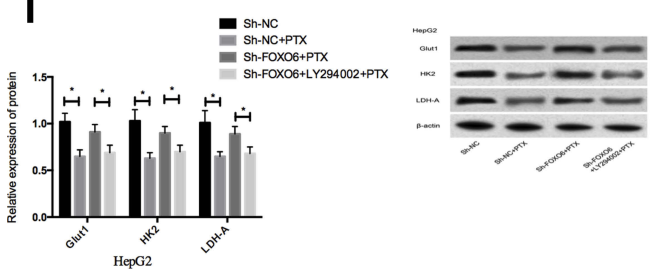

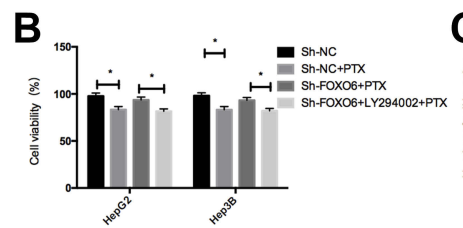

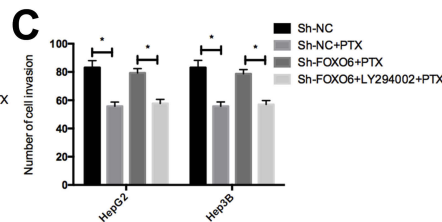

E
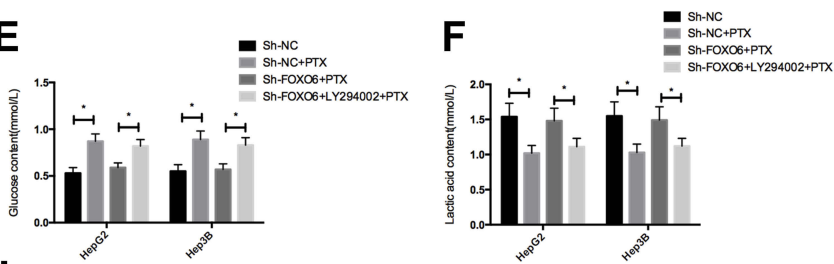

H
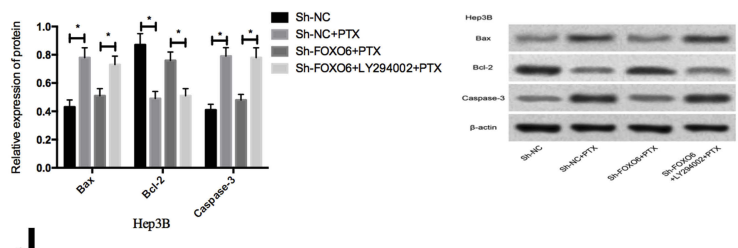

J

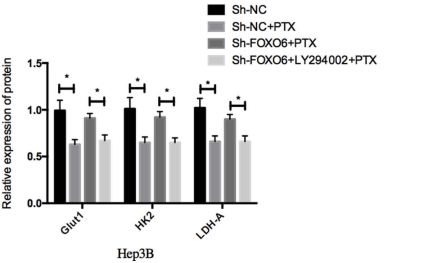

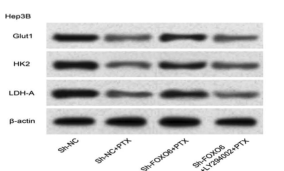

Figure 6 Effects of inhibiting PI3K/Akt signaling pathway on HCC cells after FOXO6 knockdown. (A) Expression of p-Akt and p-PI3K proteins in HCC cells after inhibiting the PI3K/Akt signaling pathway. (B) Comparison of cell viability. (C) Comparison of cell invasiveness. (D) Comparison of apoptotic rates. (E) Comparison of glucose content in the culture medium. (F) Comparison of lactic acid content in the culture medium. (G) Expression of apoptosis-related proteins in HepG2 cells. (H) Expression of apoptosis-related proteins in Hep3B cells. (I) Expression of glycolysis-related proteins in HepG2 cells. (J) Expression of glycolysis-related proteins in Hep3B cells. *Indicates P<0.05.

Metabolic reprogramming is one of the major characteristics of tumors, while glycolysis being the most important manifestation of metabolic reprogramming, playing an essential part in tumorigenesis and progress. ${ }^{22}$ Glycolysis of tumor cells can be used for energy supply to tumor cells by consuming glucose, and the generated lactic acid will be secreted outside the cell to promote the invasion and metastasis of tumor cells. ${ }^{23}$ Therefore, digging deep into the upstream molecular mechanism of glycolysis in tumors is of great significance. In this study, downregulation of FOXO6 expression was found to inhibit the glycolysis in HCC cells significantly, which was reflected by an increase of glucose content in the cell culture medium, the decrease of lactic acid, and the decrease expressions of Glut1, HK2 and LDH-A protein in cells. Glut1, HK2, and LDH-A proteins are key molecules in the glycolysis process of cancer cells, which can affect the glucose uptake and lactic acid production of cancer cells. ${ }^{24}$ However, up-regulation of FOXO6 expression can significantly promote glycolysis in HCC cells. Some studies ${ }^{25}$ suggested that FOXO6 produced a marked effect in the metabolism of cancer cells, which was validated in the present study. PI3K/Akt signaling pathway is a classical pathway in tumorigenesis and development. The current study demonstrated that down-regulation of FOXO6 expression could significantly inhibit the activation of PI3K/Akt signaling pathway and vice versa, suggesting that FOXO6 may affect glycolysis and chemotherapy resistance of HCC cells by affecting the PI3K/Akt signaling pathway.

In order to prove the effect of PI3K/Akt signaling pathway on $\mathrm{HCC}$ cells, PI3K/Akt signaling pathway was activated and intervened, and HCC cells were treated with paclitaxel to observe its effect on HCC cells. The results showed that SiFOXO6 could further enhance the inhibition of paclitaxel on the proliferation, invasion and glycolysis of $\mathrm{HepG} 2$ and Hep3B cells, and reverse the apoptosis of HepG2 and Hep3B cells induced by paclitaxel, but the promotion was inhibited after the adding of 740Y-P. On the contrary, Sh-FOXO6 could restore the inhibition of paclitaxel on the proliferation, invasion and glycolysis of HepG2 and Hep3B cells, and inhibit the apoptosis of HepG2 and Hep3B cells induced by paclitaxel, but the effect 
was also reversed after adding LY294002. This suggested that FOXO6 might affect the glycolysis and chemosensitivity of cancer cells by affecting the PI3K/Akt signaling pathway. Some studies ${ }^{26}$ reported that FOXO6 was able to affect the proliferation, invasion and glycolysis of colorectal cancer cells through the PI3K/Akt signaling pathway, which accorded with the phenotype observed in this study.

To sum up, knocking down FOXO6 expression can inhibit the proliferation, invasion and glycolysis of HCC cells by inhibiting the activation of PI3K/Akt signaling pathway, and reverse the drug resistance of HCC cells to paclitaxel, which may be a new molecular target for the treatment of HCC. However, this study still has room for improvement. For example, tumor formation experiment has not been conducted in nude mice, so the effect of FOXO6 on tumor size in nude mice after the intervention of paclitaxel remains unclear. Secondly, the upstream regulation mechanism of FOXO6 still waits to be explored. We will carry out more basic experiments to address these deficiencies to supplement and improve our conclusions.

\section{Funding}

This study was supported by Zhejiang Provincial Health Medicine Health Technology Project (2019KY663) and Wenzhou Science and Technology Project, Zhejiang Province (Y20180182).

\section{Disclosure}

The authors report no conflicts of interest in this work.

\section{References}

1. Schlesinger S, Aleksandrova K, Pischon T, et al. Abdominal obesity, weight gain during adulthood and risk of liver and biliary tract cancer in a European cohort. Int J Cancer. 2013;132(3):645-657. doi:10.1002/ ijc.v132.3

2. Jemal A, Bray F, Center MM, Ferlay J, Ward E, Forman D. Global cancer statistics. CA Cancer J Clin. 2011;61(2):69-90. doi:10.3322/caac.v61:2

3. Ryu T, Takami Y, Wada Y, Hara T, Sasaki S, Saitsu H. Efficacy of surgical microwave ablation for recurrent hepatocellular carcinoma after curative hepatectomy. HPB (Oxford). 2019. doi:10.1016/j.hpb.2019.08.001

4. Siegel R, Ma J, Zou Z, Jemal A. Cancer statistics, 2014. CA Cancer J Clin. 2014;64(1):9-29. doi:10.3322/caac.21208

5. Mollard S, Ciccolini J, Imbs DC, El Cheikh R, Barbolosi D, Benzekry S. Model driven optimization of antiangiogenics + cytotoxics combination: application to breast cancer mice treated with bevacizumab + paclitaxel doublet leads to reduced tumor growth and fewer metastasis. Oncotarget. 2017;8(14):23087-23098. doi:10.18632/oncotarget.15484

6. Pan Z, Gollahon L. Taxol directly induces endoplasmic reticulum-associated calcium changes that promote apoptosis in breast cancer cells. Breast J. 2011;17(1):56-70. doi:10.1111/j.1524-4741. 2010.00988.x

7. Accili D, Arden KC. FoxOs at the crossroads of cellular metabolism, differentiation, and transformation. Cell. 2004;117(4):421-426. doi:10. 1016/S0092-8674(04)00452-0
8. Hornsveld M, Dansen TB, Derksen PW, Burgering BMT. Reevaluating the role of FOXOs in cancer. Semin Cancer Biol. 2018;50:90-100. doi:10.1016/j.semcancer.2017.11.017

9. Wang J, Yang H, Li W, Xu H, Yang X, Gan L. Thioredoxin 1 upregulates FOXO1 transcriptional activity in drug resistance in ovarian cancer cells. Biochim Biophys Acta. 2015;1852(3):395-405. doi:10.1016/j.bbadis.2014.12.002

10. Farhan M, Wang H, Gaur U, Little PJ, Xu J, Zheng W. FOXO signaling pathways as therapeutic targets in cancer. Int J Biol Sci. 2017;13(7):815-827. doi:10.7150/ijbs.20052

11. Lee $\mathrm{S}$, Dong HH. FoxO integration of insulin signaling with glucose and lipid metabolism. J Endocrinol. 2017;233(2):R67-R79. doi:10. 1530/JOE-17-0002

12. Chen HY, Chen YM, Wu J, et al. Expression of FOXO6 is associated with oxidative stress level and predicts the prognosis in hepatocellular cancer: a comparative study. Medicine (Baltimore). 2016;95(21):e3708.

13. Lee BH, Lee DS, Cho CW, Yun SS. Role and limitation of neoadjuvant hepatic arterial infusion chemotherapy in advanced hepatocelluar carcinoma patients with child-pugh class A. World J Surg Oncol. 2019;17(1):143. doi:10.1186/s12957-019-1685-6

14. Wang L, Yang Q, Peng S, Liu X. The combination of the glycolysis inhibitor 2-DG and sorafenib can be effective against sorafenib-tolerant persister cancer cells. Onco Targets Ther. 2019;12:5359-5373. doi:10.2147/OTT.S212465

15. Psenakova K, Kohoutova K, Obsilova V, Ausserlechner MJ, Veverka V, Obsil T. Forkhead domains of FOXO transcription factors differ in both overall conformation and dynamics. Cells. 2019;8(9). doi:10.3390/cells8090966

16. Alasiri G, Jiramongkol Y, Zona S, et al. Regulation of PERK expression by FOXO3: a vulnerability of drug-resistant cancer cells. Oncogene. 2019;38(36):6382-6398. doi:10.1038/s41388-019-0890-7

17. Lallemand F, Petitalot A, Vacher S, et al. Involvement of the FOXO6 transcriptional factor in breast carcinogenesis. Oncotarget. 2018;9 (7):7464-7475. doi:10.18632/oncotarget.v9i7

18. Meena AS, Sharma A, Kumari R, Mohammad N, Singh SV, Bhat MK. Inherent and acquired resistance to paclitaxel in hepatocellular carcinoma: molecular events involved. PLoS One. 2013;8(4): e61524. doi:10.1371/journal.pone.0061524

19. van der Vos KE, Coffer PJ. The extending network of FOXO transcriptional target genes. Antioxid Redox Signal. 2011;14(4):579-592. doi:10.1089/ars.2010.3419

20. Birkenkamp KU, Coffer PJ. Regulation of cell survival and proliferation by the FOXO (forkhead box, class O) subfamily of forkhead transcription factors. Biochem Soc Trans. 2003;31(Pt 1):292-297. doi:10.1042/bst0310292

21. Hillion J, Le Coniat M, Jonveaux P, Berger R, Bernard OA. AF6q21, a novel partner of the MLL gene in $\mathrm{t}(6 ; 11)(\mathrm{q} 21 ; \mathrm{q} 23)$, defines a forkhead transcriptional factor subfamily. Blood. 1997;90 (9):3714-3719. doi:10.1182/blood.V90.9.3714

22. Tekade RK, Sun X. The warburg effect and glucose-derived cancer theranostics. Drug Discov Today. 2017;22(11):1637-1653. doi:10.1016/j.drudis.2017.08.003

23. Lu Y, Wang L, Ding W, et al. Ammonia mediates mitochondrial uncoupling and promotes glycolysis via HIF-1 activation in human breast cancer MDA-MB-231cells. Biochem Biophys Res Commun. 2019;519(1):153-159. doi:10.1016/j.bbrc.2019.08.152

24. Liu L, Lei B, Wang L, et al. Protein kinase C-iota-mediated glycolysis promotes non-small-cell lung cancer progression. Onco Targets Ther. 2019;12:5835-5848. doi:10.2147/OTT.S207211

25. Sakamaki J, Daitoku H, Yoshimochi K, Miwa M, Fukamizu A. Regulation of FOXO1-mediated transcription and cell proliferation by PARP-1. Biochem Biophys Res Commun. 2009;382(3):497-502. doi:10.1016/j.bbrc.2009.03.022

26. Li Q, Tang H, Hu F, Qin C. Silencing of FOXO6 inhibits the proliferation, invasion, and glycolysis in colorectal cancer cells. J Cell Biochem. 2019;120(3):3853-3860. doi:10.1002/jcb.v120.3 


\section{Publish your work in this journal}

OncoTargets and Therapy is an international, peer-reviewed, open access journal focusing on the pathological basis of all cancers, potential targets for therapy and treatment protocols employed to improve the management of cancer patients. The journal also focuses on the impact of management programs and new therapeutic agents and protocols on patient perspectives such as quality of life, adherence and satisfaction. The manuscript management system is completely online and includes a very quick and fair peer-review system, which is all easy to use. Visit http://www.dovepress.com/ testimonials.php to read real quotes from published authors. 